Pacific Journal of Mathematics

ON A CLASS OF TOPOLOGICAL GROUPS MORE GENERAL 


\title{
ON A CLASS OF TOPOLOGICAL GROUPS MORE GENERAL THAN SIN GROUPS
}

\author{
R. W. BAgley, T. S. WU AND J. S. YANG
}

\begin{abstract}
We consider a class of topological groups more general than those with small invariant nieghborhoods of the identity, SIN-groups. We refer to these more general groups as $\boldsymbol{N}$-groups. We prove that a compactly generated $N$-group is a $\mathrm{SIN}$-group. This result has several applications, including the following: A locally compact $N$-group is unimodular.
\end{abstract}

Introduction. There has been considerable interest in topological groups with small invariant neighborhoods of the identity. There is a very good bibliography of the literature on these groups in [6]. In this paper, we are interested in a more general class of groups which share some of the interesting properties of SIN-groups. We obtain some of these properties and attempt to determine which of the more general class are SIN-groups.

If $G$ is a topological group and $\mathscr{B}$ is a subgroup of the group of topological automorphisms of $G$, we say that $G$ is an $N(\mathscr{B})$-group or simply $G$ is $N(\mathscr{B})$ if the following holds: For each pair of nets $\left\{x_{\alpha}\right\}$ in $G$ and $\left\{\phi_{\alpha}\right\}$ in $\mathscr{B}$ such that $\left\{x_{\alpha}\right\}$ converges to the identity, the net $\left\{\phi_{\alpha}\left(x_{\alpha}\right)\right\}$ converges to the identity or fails to converge. We say that $G$ is $\operatorname{SIN}(\mathscr{B})$ if $G$ has small neighborhoods of the identity which are invariant under the elements of $\mathscr{B}$. This is tantamount to saying that for any net $\left(\phi_{\alpha}, x_{\alpha}\right) \in \mathscr{B}$ $\times G$ with $x_{\alpha} \rightarrow e$ we have $\phi_{\alpha}\left(x_{\alpha}\right) \rightarrow e$. If $\mathscr{B}$ is the group of all inner automorphisms of $G$ we use " $N$ " and "SIN" for " $N(\mathscr{B})$ " and "SIN $(\mathscr{B})$ " respectively.

One of our most useful results is the following: If $\mathscr{B}$ is a completely generated group of automorphisms of $G$ in an admissible topology and $G$ is a locally compact $N(\mathscr{B})$-group, then $G$ is $\operatorname{SIN}(\mathscr{B})$. We use this result to prove that a locally compact $N$-group is unimodular, Theorem 4 ; a result on invariant measures, Corollary 4; and a result on semidirect products, Proposition 3. We prove a structure theorem for locally compact totally disconnected $N$-groups and construct an example of a locally compact $N$-group which cannot be embedded in a locally compact SIN-group. This solves a problem posed in [6] in the category of locally compact groups. In 
a subsequent paper we apply some of the present results to pro-Lie groups.

For relations between SIN-groups and MAP groups see [2], in particular Theorem 2.9 and [6].

We will use the symbol " $\mathscr{B}$ " to denote an arbitrary group of topological automorphisms of a group $G$ unless otherwise specified. We note that a locally compact group $G$ is $N(\mathscr{B})$ if and only if, for each neighborhood $U$ of the identity and each compact set $C$, there is a neighborhood $V$ of the identity such that $\phi(V) \subset U \subset(G-C)$ for every $\phi \in \mathscr{B}$.

Proposition 1. If $G$ is a locally connected, locally compact $N(\mathscr{B})$-group, then $G$ is $\operatorname{SIN}(\mathscr{B})$.

Proof. Let $U$ be a compact neighborhood of the identity. Let $U^{\prime}$ be an open neighborhood of the identity contained in $U$. Since $G$ is locally connected and is an $N(\mathscr{B})$-group, there is a connected neighborhood $V$ of the identity such that $\mathscr{B}(V) \subset U^{\prime} \cup(G-U)$. Since each $\phi(V)$ is connected and contains $e, \mathscr{B}(V)$ is connected and hence contained in $U^{\prime} \subset U$.

If $\mathscr{B}$ is a group of automorphisms of $G$ and $K$ is a normal subgroup of $G$ which is invariant under the elements of $\mathscr{B}$, we let $\tilde{\mathscr{B}}$ represent the automorphisms of $G / K$ induced by the elements of $\mathscr{B}$ in the natural way.

Proposition 2. If $G$ is a locally compact $N(\mathscr{B})$-group, then the following hold:

(i) $G / K$ is $N(\tilde{\mathscr{B}})$.

(ii) $G$ is $\operatorname{SIN}(\mathscr{B})$ if and only if $G / K$ is $\operatorname{SIN}(\tilde{\mathscr{B}})$.

Proof of (i). Let $C$ be a compact subset of $G$ such that $C K=C$ and $U$ a $K$-saturated neighborhood of $e$ in $G$. Then, since $G$ is an $N(\mathscr{B})$-group there is a neighborhood $V$ of $e$ such that $C \cap \mathscr{B}(V) \subset U$. It follows that $C / K \cap \tilde{\mathscr{B}}(V K / K) \subset U / K$ and the proof of (i) is complete.

Proof (ii). Clearly $G / K$ is $\operatorname{SIN}(\tilde{\mathscr{B}})$ if $G$ is $\operatorname{SIN}(\mathscr{B})$. To prove the other implication, let $G / K$ be $\operatorname{SIN}(\tilde{\mathscr{B}})$. Let $U$ be a compact neighborhood of the identity. We let $C=K U$. Since $G / K$ is $\operatorname{SIN}(\tilde{\mathscr{B}})$, there is a neighborhood $W$ of the identity in $G$ such that $W K=K W$ and $B(W) \subset C$. But since $G$ is $N(\mathscr{B})$ there is a neighborhood $V$ of the identity such that $V \subset W$ and $C \cap \mathscr{B}(V) \subset U$. But $\mathscr{B}(V) \subset \mathscr{B}(W) \subset C$, whence $\mathscr{B}(V) \subset U$.

THEOREM 1. If $G$ is a locally compact $N(\mathscr{B})$-group such that $G / G_{0}$ is compact, then $G$ is $\operatorname{SIN}(\mathscr{B})$. 
Proof. Let $K$ be the maximal compact normal subgroup of $G$ such that $G / K$ is a Lie group (see 3.1, p. 180 [3]). By Proposition 2(i), $G / K$ is an $N(\tilde{\mathscr{B}})$-group and by Proposition $1, G / K$ is $\operatorname{SIN}(\tilde{\mathscr{B}})$. The proof is completed on applying (ii) of Proposition 2.

THEOREM 2. If $\mathscr{B}$ is a compactly generated group of automorphisms of $G$ in an admissible topology and $G$ is a locally compact $N(\mathscr{B})$-group, then $G$ is $\operatorname{SIN}(\mathscr{B})$.

Proof. Let $U$ be a compact neighborhood of the identity of $G$. We obtain a neighborhood $V$ of the identity such that $\mathscr{B}(V) \subset U$. Let $\mathscr{C}$ be a symmetric compact generating set of $\mathscr{B}$. There is a neighborhood $W$ of the identity of $G$ such that $\mathscr{C}(W) \subset U$. Since $G$ is an $N(\mathscr{B})$-group, there is a neighborhood $V$ of the identity such that $U \cap \mathscr{B}(V) \subset W$ and $\mathscr{C}(V) \subset W$. By induction $\mathscr{C}^{n}(V) \subset W$. First, $\mathscr{C}$ and $V$ were chosen so that this holds when $n=1$. If $\mathscr{C}^{n}(V) \subset W$, then $\mathscr{C}^{n+1}(V) \subset \mathscr{C}(W) \subset U$, whence $\mathscr{C}^{n+1}(V) \subset U \cap \mathscr{B}(V) \subset W$. This completes the induction. It follows that $\mathscr{B}(V)=\bigcup_{n} \mathscr{C}^{n}(V) \subset W \subset U$ as desired.

In the following we denote the center of $G$ by " $Z$ ".

COROLlary 1. If $G$ is a locally compact $N$ group and $H$ is a normal subgroup of $G$ such that $G / H \cap Z$ is compactly generated, then $G$ has small $H$-invariate neighborhoods of the identity.

Proof. Let $\mathscr{B}$ be the group of inner automorphisms of $G$ determined by the elements of $H$. Then $G$ is a locally compact $N(\mathscr{B})$ group and $\mathscr{B}$ is compactly generated in the compact-open topology. By Theorem $2, G$ is $\operatorname{SIN}(\mathscr{B})$, which is the desired conclusion.

COROLlaRY 2. If $\mathscr{B}$ is compactly generated in an admissible topology and $G$ is a locally compact $N(\mathscr{B})$-group, then $G$ is $\mathscr{B}$-unimodular.

Proof. By Theorem 2, $G$ is $\operatorname{SIN}(\mathscr{B})$ and hence $\mathscr{B}$-unimodular by Proposition 2.4 [2].

The following is a special case of Corollary 1 of Theorem 2. We state it as a theorem because of its frequent subsequent use.

THEOREM 3. If $G$ is a locally compact $N$-group such that $G / Z$ is compactly generated, then $G$ is SIN. 
THEOREM 4. If $G$ is a locally compact $N$-group, then $G$ is unimodular.

Proof. Let $g$ be an element of $G$ and $V$ a compact neighborhood of the identity. Let $H$ be the open subgroup generated by $g V \cup V$. By Theorem $3, H$ is SIN. Thus $H$ is unimodular and it follows from Proposition 20, page 86 [5] that $G$ is unimodular.

A subgroup $H$ is uniform if $H$ is closed and $G / H$ is compact.

COROLlaRY 4. If $G$ is a locally compact, $\sigma$-compact group such that $G=F H$ where $F$ is a normal $N$-subgroup and $H$ is a uniform subgroup, then $G / H$ admits a finite invariant measure.

Proof. Since $G$ is $\sigma$-compact the map $\theta: F / F \cap H \rightarrow G / H$ defined by $\theta(f F \cap H)=f H$ for $f \in F$ is a homeomorphism. By Theorem 4, the modular functions for $F$ and $F \cap H$ correspond on $F \cap H$. Thus $F / F \cap H$ admits a finite invariant measure and the conclusion follows from Lemma $1.7[8]$.

We are now able to give an easy proof of a result of S. P. Wang, Lemma 3.3 [9].

THEOREM ( $S$. P. Wang). If $G$ is a locally compact group, $H$ is a uniform subgroup, and $G$ contains an open normal MAP subgroup $F$, then $G / H$ admits a finite invariant measure.

Proof. Since $F$ is open and normal, the natural mapping of $F / F \cap H$ onto $\mathrm{FH} / \mathrm{H}$ is a homeomorphism. By Corollary 4 to Theorem $4, F H / H$ admits a finite invariant measure. Now $G / F H$ is finite since $F H$ is open and $H$ is a uniform subgroup. Thus $G / H$ admits a finite invariant measure.

Among the examples of semi-direct products of two SIN groups which are not SIN are the following: The circle group acting on the plane by rotation and the discrete cyclic group acting on the compact group of bisequences by shifts. We consider a semi-direct product $G \times{ }_{\eta} H$ defined by the homomorphism $\eta: H \rightarrow \mathscr{A}$, the group of automorphisms of $G$. We denote the kernel of $\eta$ by $K_{\eta}$. The group operation in $G \times{ }_{\eta} H$ is defined as follows: $(g, h)\left(g_{1}, h_{1}\right)=\left(g \eta(h)\left(g_{1}\right), h h_{1}\right)$.

Proposition 3. If $H$ is a locally compact, locally connected group, $G$ is a locally compact $\mathrm{SIN}$-group, $\mathrm{H} / K_{\eta}$ is compactly generated, and $\mathrm{G} \times{ }_{\eta} \mathrm{H}$ is an $N$-group, then $G \times{ }_{\eta} H$ is $\mathrm{SIN}$. 
Proof. We first show that $G$ is $\operatorname{SIN}(\eta(H))$. Let $U \times V$ be a neighborhood of the identity $\left(e_{1}, e_{2}\right)$ of $G \times{ }_{\eta} H$ and let $C$ be any compact subset of $G$. Since $G \times{ }_{\eta} H$ is an $N$-group, there is a neighborhood $W$ of the identity of $G$ such that

$$
\left(\eta(h)(x), e_{2}\right)=\left(e_{1}, h\right)\left(x, e_{2}\right)\left(e_{1}, h^{-1}\right) \in U \times V \cup\left(G \times H-C \times e_{2}\right)
$$

whenever $x \in W$. Thus $\eta(h)(x) \in U \cup(G-C)$. It follows that $G$ is $N(\eta(H))$; and since $h / K_{\eta}$ is compactly generated, $G$ is $\operatorname{SIN}(\eta(H))$ by Theorem 2. To complete the proof we let $U_{1} \times U_{2}$ be an open neighborhood of the identity of $G \times{ }_{\eta} H$ and let $C$ be a compact subset $G \times H$ such that $U_{1} \times U_{2} \subset C$. Since $G$ is SIN there is a neighborhood $U$ of the identity of $G$ such that $g U g^{-1} \subset U_{1}$. Since $G \times{ }_{\eta} H$ is an $N$-group, there is a neighborhood $W$ of the identity of $G$ and a connected neighborhood $V$ of the identity of $H$ such that

$$
(g, h) W \times V(g, h)^{-1} \subset U_{1} \times U_{2} \cup(G \times H-C)
$$

and

$$
\eta(h)(W) \subset U
$$

If $w \in W$, then

$$
\begin{aligned}
(g, h)\left(w, e_{2}\right)(g, h)^{-1} & =(g \eta(h)(w), h)\left(\eta\left(h^{-1}\right)\left(g^{-1}\right), h^{-1}\right) \\
& =\left(g \eta(h)(w) \eta(h)\left(\eta\left(h^{-1}\right)\left(g^{-1}\right)\right), e_{2}\right) \\
& =\left(g \eta(h)(w) g^{-1}, e_{2}\right) \in U_{1} \times e_{2} .
\end{aligned}
$$

Thus $(g, h) w \times V(g, h)^{-1}$ intersects $U_{1} \times U_{2}$ for every $(g, h)$. It follows that $(g, h) w \times V(g, h)^{-1} \subset U_{1} \times U_{2}$ for every $(g, h) \in G \times H$ and $w \in$ $W$ since $V$ is connected. This completes the proof of Proposition 3.

To see the necessity of the hypothesis of local connectedness we point to Example 2 below, a semi-direct product $H \times_{\eta} K$ which is a locally compact MAP group hence $N$ but not SIN, even though $K$ is compact and $H$ is discrete normal.

EXAMPLE 1 . We construct a locally compact $N$ group which is not embeddable in a locally compact SIN group. Let $F$ be a discrete group which is not MAP. For each positive integer $n$ we let $H_{n}=F \times F$ and $K_{n}=Z_{2}$. Let $H=\sum H_{n}$, the direct sum with discrete topology and $K=\Pi K_{n}$, the direct product with product topology. We define the semi-direct product $H \times{ }_{\eta} K$ as follows: If the $i$ th coordinate of $k$ is 1 , 
then $\eta(k)(h)$ has $i$ th coordinate $\left(f_{2}, f_{1}\right)$ where $\left(f_{1}, f_{2}\right)$ is the $i$ th coordinate of $h$. If the $i$ th coordinate of $k$ is 0 , then the $i$ th coordinate of $\eta(k)(h)$ is unchanged. It is a routine computation to show that $H \times{ }_{\eta} K=$ $G$ is an $N$ group. As a matter of fact, if $H$ is given the product topology, the corresponding group is SIN. Thus $G$ is embeddable in a nonlocally compact SIN group. By "embedding" we mean a continuous isomorphism.

To see that $G$ is not embeddable in a locally compact SIN group, we note that if it were, it would contain an open normal MAP subgroup. It is easy to see that any open normal subgroup contains a subgroup isomorphic to the direct sum of all but a finite number of the groups $H_{n}$. This contradicts the fact that $F$ is not MAP.

The following is an example of an MAP group which is not SIN and as mentioned above shows the necessity of the hypothesis or local connectedness in Proposition 3.

EXAMPLE 2. For each positive integer $i$ let $H_{l}=Z_{3}$ and $K_{l}=Z_{2}$. Let $H$ be the direct sum $\sum H_{l}$ with discrete topology and $K$ be the direct product $\Pi K$, with product topology. Let $G=H \times{ }_{\eta} K$ defined as follows: The $i$ th coordinate of $\eta(k)(h)$ is the $i$ th coordinate of $h$ if the corresponding coordinate of $k$ is 0 . Otherwise, the $i$ th coordinate of $\eta(k)(h)$ is the negative of the $i$ th coordinate of $h$. The group $G$ is MAP since $H \times{ }_{\eta} K$ with the product topology is compact.

Since any topological group $G$ is a quotient of the free topological group generated by the underlying space of $G$ and since every free topological group is MAP [6], quotients of $N$ groups are not necessarily $N$-groups. In Proposition 2 we say that, if a compact normal subgroup is factored from an $N$-group, the result is an $N$ group.

LEMMA 1. If $G$ is a locally compact totally disconnected $N$ group and $X$ is a compact subset of $G$, there is an arbitrarily small compact open subgroup which is normal in some open subgroup containing $X$.

Proof. Let $H$ be the open subgroup generated by $X$ and some compact neighborhood of the identity. By Theorem 3, $H$ is SIN and therefore contains arbitrarily small compact open normal subgroups.

Proposition 4. If $G$ is a totally disconnected locally compact $N$ group and $A$ is a closed normal central subgroup which has no non-trivial bounded elements, then $G / A$ is an $N$ group. 
Proof. Suppose $\left\{a_{\alpha} g_{\alpha} u_{\alpha} g_{\alpha}^{-1}\right\}$ converges to $x$ where $a_{\alpha} \in A$ and $\left\{u_{\alpha}\right\}$ converges to $e$. That is $g_{\alpha} u_{\alpha} g_{\alpha}^{-1} A$ converges to $x A$ in $G / A$. Let $K$ be a compact open subgroup such that $x K=K x$ by Lemma 1 . We can choose $\alpha_{0}$ such that $u_{\alpha} \in K$ and $a_{\alpha} g_{\alpha} u_{\alpha} g_{\alpha}^{-1} \in x K$ for all $\alpha \geq \alpha_{0}$. Since the sequence $\left\{g_{\alpha} u_{\alpha}^{n} g_{\alpha}^{-1}\right\}$ is frequently in $K$ for each $\alpha \geq \alpha_{0}$, for any fixed $\alpha \geq \alpha_{0}$, there is an integer $n$ such that $a_{\alpha}^{n}$ and $a_{\alpha_{0}}^{n}$ are in $x^{n} K$. Thus $\left(a_{\alpha_{0}}^{-1} a_{\alpha}\right)^{n} \in K$. This implies that $a_{\alpha_{0}}^{-1} a_{\alpha}=e$ since $A$ has no non-trivial compact elements. It follows that $\left\{g_{\alpha} u_{\alpha} g_{\alpha}^{-1}\right\}$ converges to $a_{\alpha_{0}}^{-1} x$ and since $G$ is an $N$ group, $x=a_{\alpha_{0}} \in A$. This completes the proof.

For a topological group $G$ we denote by $P(G)$ the periodic elements of $G$ and by $B(G)$ the bounded elements of $G$.

Proposition 5. If $G$ is a locally compact totally disconnected $N$ group, $F$ is a closed-normal SIN subgroup, and for some open compact normal subgroup $K$ of $F, B(F / K)$ is compactly generated, then $F$ has small $G$ invariant neighborhoods of the identity.

Proof. Since $B(F / K)=B(F) / K$ and $K$ is compact, $B(F)$ is compactly generated. By $3.22[2], P(F) \cap B(F)$ is compactly generated and therefore compact by 3.17 [2]. By Theorem 3 [11] and Corollary 5.6 [10], $P(F) \cap B(F)$ is open and characteristic in $F$. Thus, if a net $\left\{x_{\alpha}\right\}$ in $F$ converges to the identity and $\left\{g_{\alpha}\right\}$ is any net in $G$, the net $\left\{g_{\alpha} x_{\alpha} g_{\alpha}^{-1}\right\}$ is eventually in $P(F) \cap B(F)$ and consequently has a subnet which converges. This subnet converges to the identity since $G$ is an $N$ group. This completes the proof.

COROLlary 5. If $G$ is a locally compact totally disconnected $N$ group and $F$ is a closed-normal SIN subgroup such that $B(F)$ is compactly generated, then $F$ has small $G$ invariant neighborhoods of the identity.

Proof. Since $F$ has compact open-normal subgroups, the corollary follows immediately.

Since almost connected as well as compactly generated $N$ groups are SIN, there are structure theorems for such groups [2]. The following theorem is a structure theorem for totally disconnected $N$ groups.

THEOREM 5. If $G$ is a second countable locally compact totally disconnected $N$ group which is not SIN and $K$ is a compact open subgroup there are sequences of open subgroups $\left\{G_{n}\right\}$ and $\left\{K_{n}\right\}$ such that $\left\{G_{n}\right\}$ is strictly monotone increasing, $\left\{K_{n}\right\}$ is strictly monotone decreasing and

1. Each $G_{n}$ is a compactly generated SIN group, $G=\bigcup G_{n}$, and $K_{n}$ is a maximal compact open normal subgroup of $G_{n}$ contained in $K$. 
2. The group of inner automorphisms of $G$ restricted to $L=\cap K_{n}$ is equicontinuous on $L$.

3. $G_{n} / L=\lim _{i \geq n} G_{n} / K_{i}$.

Proof. There is a sequence $\left\{g_{i}\right\}$ in $G$ such that $G$ is generated by $\left\{K, g_{1}, g_{2}, \ldots\right\}$. Let $G_{1}$ be the open subgroup generated by $\left\{K, g_{1}\right\}$ and let $K_{1}$ be the maximal compact open subgroup of $G_{1}$. For some integer $n_{1}$, the open subgroup $G_{2}$ generated by $\left\{K, g_{1}, \ldots, G_{n_{1}}\right\}$ contains a maximal compact open normal subgroup $K_{2}$ properly contained in $K_{1}$. The strict inclusion is obtained since otherwise $K$ would be normal in $G$ contradicting the fact that $G$ is not SIN. An $N$ group with a compact open normal subgroup is clearly SIN. By induction we obtain $G_{n}$ and $K_{n}$ for each positive integer $n$ with the desired properties. The first conclusion is obvious by construction and the fact that any compactly generated $N$ group is SIN. The second conclusion follows since $L$ is compact and normal in $G$ and $G$ is an $N$ group. For the third conclusion we note that, if $U$ is any compact neighborhood of the identity of $G$, then some $K_{i}$ is contained in $U L$.

We note that, since $L$ is compact and normal in $G$ and $G$ is not SIN, then $L$ is not open. Also, since there is a sequence of compact open subgroups which forms a neighborhood base at the identity of $G$, there is a monotone decreasing sequence of compact normal subgroups $\left\{L_{n}\right\}$ such that $G=\lim _{\leftarrow} G / L_{n}$. Each $L_{n}$ is obtained in the manner above.

Added in proof. There is a gap in the description of Example 1. The problem is resolved in the paper: Maximal compact normal subgroups and pro-Lie groups by R. W. Bagley and T. S. Wu to appear in Proc. Amer. Math. Soc.

\section{REFERENCES}

[1] R. W. Bagley, T. S. Wu, and J. S. Yang, A note on pro-Lie groups, to appear in Trans. Amer. Math. Soc.

[2] S. Grosser and M. Moskowitz, Compactness conditions in topological groups, J. Reine Angew. Math., 246 (1971), 1-40.

[3] G. Hochschild, The Structure of Lie Groups, Holden-Day, Inc., San FranciscoLondon-Amsterdam (1965).

[4] K. Iwasawa, On some types of typological groups, Annals of Math., 50 (1949), $507-558$.

[5] L. Lachbin, The Haar Integral, Princeton (1965).

[6] E. T. Ordman and S. A. Morris, Almost lecally invariant topological groups, J. London Math. Soc., 9 (1974), 30-34. 
[7] T. W. Palmer, Classes of nonabelian, noncompact, locally compact groups, Rocky Mountain J. Math., 8 (1978), 683-741.

[8] M. S. Raghunathan, Discrete subgroups of Lie groups, Ergebnisse der Mathematik und ihrer Grenzgebiete, Berlin-Heidelberg-New York (1972).

[9] S. P. Wang, On density properties of certain subgroups of locally compact groups, Duke Math. J., 43 (1976), 561-578.

[10] S. P. Wang, Compactness properties of topological groups, Trans. Amer. Math. Soc., 15 (1971), 301-314.

[11] T. S. Wu and Y. K. Yu, Compactness properties of topological groups, Michigan Math. J., 19 (1972), 299-313.

Received June 17, 1983 and in revised form October 3, 1983. The authors would like to thank the referee for his valuable suggestions which in several cases led to much more readable proofs.

UNIVERSITY OF MIAMI

Coral Gables, FL 33124

CASE WASTERN RESERVE UNIVERSITY

Cleveland, OH 44106

AND

UNIVERSity OF SOUTH CAROLINA

Columbia, SC 29208 



\section{PACIFIC JOURNAL OF MATHEMATICS EDITORS}

DONALD BABBITT (Managing Editor)
University of California
Los Angeles, CA 90024
CHARLES R. DEPrIMA
California Institute of Technology
Pasadena, CA 91125
R. FINN
Stanford University
Stanford, CA 94305

\author{
HERMANN FLASCHKa \\ University of Arizona \\ Tucson, AZ 85721 \\ RAMESH A. GANGOLLI \\ University of Washington \\ Seattle, WA 98195 \\ ROBION KIRBY \\ University of California \\ Berkeley, CA 94720 \\ C. C. Moore \\ University of California \\ Berkeley, CA 94720
}

Hugo Rossi

University of Utah

Salt Lake City, UT 84112

H. SAMELSON
Stanford University
Stanford, CA 94305

HAROLD STARK

University of California, San Diego

La Jolla, CA 92093

\section{ASSOCIATE EDITORS}
R. ARens
E. F. BECKENBACH (1906-1982)
B. H. NeUmanN
F. WOLF
K. YOSHIDA

\begin{tabular}{ll}
\multicolumn{2}{c}{ SUPPORTING INSTITUTIONS } \\
UNIVERSITY OF ARIZONA & UNIVERSITY OF OREGON \\
UNIVERSITY OF BRITISH COLUMBIA & UNIVERSITY OF SOUTHERN CALIFORNIA \\
CALIFORNIA INSTITUTE OF TECHNOLOGY & STANFORD UNIVERSITY \\
UNIVERSITY OF CALIFORNIA & UNIVERSITY OF HAWAII \\
MONTANA STATE UNIVERSITY & UNIVERSITY OF TOKYO \\
UNIVERSITY OF NEVADA, RENO & UNIVERSITY OF UTAH \\
NEW MEXICO STATE UNIVERSITY & WASHINGTON STATE UNIVERSITY \\
OREGON STATE UNIVERSITY & UNIVERSITY OF WASHINGTON
\end{tabular}

The Supporting Institutions listed above contribute to the cost of publication of this Journal, but they are not owners or publishers and have no responsibility for its content or policies.

\footnotetext{
Mathematical papers intended for publication in the Pacıfic Journal of Mathematıcs should be in typed form or offset-reproduced (not dittoed), double spaced with large margins. Please do not use built up fractions in the text of the manuscript. However, you may use them in the displayed equations. Underline Greek letters in red, German in green, and script in blue. The first paragraph must be capable of being used separately as a synopsis of the entire paper. In particular it should contain no bibliographic references. Please propose a heading for the odd numbered pages of less than 35 characters. Manuscripts, in triplicate, may be sent to any one of the editors. Please classify according to the scheme of Math. Rev ews, Index to Vol. 39. Supply name and address of author to whom proofs should be sent. All other communications should be addressed to the managing editor, or Elaine Barth, University of California, Los Angeles, California 90024.

There are page-charges associated with articles appearing in the Pacific Journal of Mathematics. These charges are expected to be paid by the author's University, Government Agency or Company. If the author or authors do not have access to such Institutional support these charges are waived. Single authors will receive 50 free reprints; joint authors will receive a total of 100 free reprints. Additional copies may be obtained at cost in multiples of 50 .
}

The Pacific Journal of Mathematics is issued monthly as of January 1966. Regular subscription rate: $\$ 190.00$ a year (5 Vols., 10 issues). Special rate: $\$ 66.00$ a year to individual members of supporting institutions.

Subscriptions, orders for numbers issued in the last three calendar years, and changes of address should be sent to Pacific Journal of Mathematics, P.O. Box 969, Carmel Valley, CA 93924, U.S.A. Old back numbers obtainable from Kraus Periodicals Co., Route 100, Millwood, NY 10546.

The Pacific Journal of Mathematics at P.O. Box 969, Carmel Valley, CA 93924 (ISSN 0030-8730) publishes 5 volumes per year. Application to mail at Second-class postage rates is pending at Carmel Valley, California, and additional mailing offices. Postmaster: Send address changes to Pacific Journal of Mathematics, P.O. Box 969, Carmel Valley, CA 93924.

PUBLISHED BY PACIFIC JOURNAL OF MATHEMATICS, A NON-PROFIT CORPORATION Copyright $\odot 1985$ by Pacific Journal of Mathematics 


\section{Pacific Journal of Mathematics}

\section{Vol. 117, No. 2 \\ February, 1985}

Robert Walter Bagley, Ta-Sun Wu and J. S. Yang, On a class of topological groups more general than SIN groups ............... 209

Bruce Alan Barnes, Algebraic elements of a Banach algebra modulo an ideal ...............................................219

Howard D. Fegan and Peter Gilkey, Invariants of the heat equation .......233

Erica Flapan, Necessary and sufficient conditions for certain homology

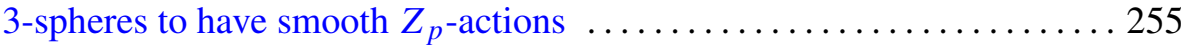

Philip R. Heath, Product formulae for Nielsen numbers of fibre maps . . . . 267

Derbiau Frank Hsu and A. Donald Keedwell, Generalized complete mappings, neofields, sequenceable groups and block designs. II ...... 291

Taqdir Husain, Orthogonal primitive idempotents and Banach algebras

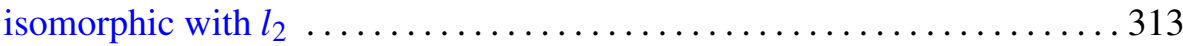

Gary M. Lieberman, Regularized distance and its applications . . . . . . . 329

William W. Menasco, Determining incompressibility of surfaces in alternating knot and link complements ............................ 353

Benjamin Muckenhoupt, Weighted reverse weak type inequalities for the Hardy-Littlewood maximal function $\ldots \ldots \ldots \ldots \ldots \ldots \ldots \ldots \ldots \ldots \ldots \ldots$

John Dacey O'Neill, Direct summands of direct products of slender

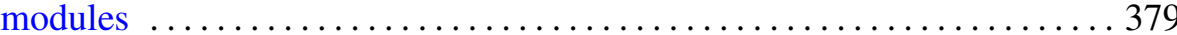

Andreas Rapp, Elimination of Malitz quantifiers in stable theories 387

Francisco José Ruiz, A unified approach to Carleson measures and $A_{p}$ weights

Hanamatagouda Pandappa Sankappanavar, Heyting algebras with dual

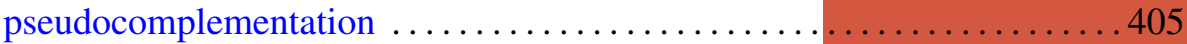

Annette Sinclair, Strong Carleman and strong uniform approximation . . . . 417

Masaaki Suzuki, The generalized Schwarz lemma for the Bergman metric

Brian Thorpe and Ludwig Tomm, Universal approximation by regular

weighted means 